Original article

\title{
Neurocognition and functional outcome in patients with psychotic, non-psychotic bipolar I disorder, and schizophrenia. A five-year follow-up
}

\author{
Estela Jiménez-López ${ }^{\mathrm{a}, \mathrm{b}, \mathrm{c}}$, Eva María Sánchez-Morla ${ }^{\mathrm{b}, \mathrm{d}, \mathrm{e}}$, Ana López-Villarreal $^{\mathrm{a}}$, \\ Ana Isabel Aparicio ${ }^{\mathrm{a}, \mathrm{b}}$, Vicente Martínez-Vizcaíno ${ }^{\mathrm{c}, \mathrm{f}}$, Eduard Vieta $^{\mathrm{b}, \mathrm{g}}$, \\ Roberto Rodriguez-Jimenez ${ }^{\mathrm{b}, \mathrm{d}, \mathrm{e}}$, José Luis Santos ${ }^{\mathrm{a}, \mathrm{b}, *}$ \\ a Department of Psychiatry, Hospital Virgen de La Luz, Cuenca, Spain \\ ${ }^{\mathrm{b}}$ CIBERSAM (Biomedical Research Networking Centre in Mental Health), Spain \\ ${ }^{\mathrm{c}}$ Universidad de Castilla-La Mancha, Health and Social Research Center, Cuenca, Spain \\ d Instituto de Investigación Sanitaria Hospital, 12 de Octubre (imas12), Madrid, Spain \\ e CogPsy-Group, Universidad Complutense de Madrid (UCM), Spain \\ ${ }^{\mathrm{f}}$ Universidad Autónoma de Chile, Facultad de Ciencias de la Salud, Talca, Chile \\ ${ }^{\mathrm{g}}$ Department of Psychiatry, Hospital Clinic of Barcelona, University of Barcelona, IDIBAPS, Barcelona, Spain
}

\section{A R T I C L E I N F O}

\section{Article history:}

Received 4 October 2018

Received in revised form 18 November 2018

Accepted 20 November 2018

Available online 28 November 2018

\section{Keywords:}

Bipolar disorder

Schizophrenia

Psychosis

Neurocognition

Psychosocial functioning

Follow-up

\begin{abstract}
A B S T R A C T
Background: Bipolar disorder (BD) and schizophrenia (SZ) are characterized by neurocognitive and functional deficits with marked heterogeneity. It has been suggested that BD with a history of psychotic symptoms (BD-P) could constitute a phenotypically homogeneous subtype characterized by greater neurocognitive and functional impairments, or by a distinct trajectory of such deficits. The aim of this study was to compare the neurocognitive and functional course of euthymic BD-P, euthymic BD patients without a history of psychosis (BD-NP), stabilized patients with schizophrenia and healthy subjects, during a five-year follow-up.

Methods: Neurocognitive and psychosocial function was examined in 100 euthymic patients with BD (50 BD-P, 50 BD-NP), 50 stabilized patients with schizophrenia (SZ), and 51 healthy controls (HC) at baseline (T1), and after a 5-year follow-up (T2).

Results: The course of both neurocognitive performance and functional outcome of patients with SZ and $\mathrm{BD}$ (BD-P and BD-NP) is stable. The profile of neurocognitive impairment of patients with SZ or BD (BD-P and BD-NP), is similar, with only quantitative differences circumscribed to certain domains, such as working memory. The subgroup of patients with BD-NP does not show functional deterioration. Conclusions: We have not found evidence of progression in the neurocognitive or psychosocial impairment in any of the three groups of patients, although it cannot be dismissed the possibility of a subset of patients with a progressive course. Other longitudinal studies with larger samples and longer duration are necessary to confirm these findings.
\end{abstract}

(c) 2018 Elsevier Masson SAS. All rights reserved.

\section{Introduction}

Bipolar disorder (BD) is characterized by neurocognitive [1,2] and functional $[3,4]$ deficits which are a core feature of the disorder. However, a marked clinical $[5,6]$, neurocognitive $[7,8]$ or functional $[9,10]$ heterogeneity has been reported, in a way that, at one end, some patients with BD seem to reach a level of

\footnotetext{
* Corresponding author at: Department of Psychiatry, Hospital Virgen de La Luz, C/Hermandad Donantes de Sangre 1, 16002, Cuenca, Spain.

E-mail address: jlsantosg@sescam.jccm.es (J.L. Santos).
}

psychosocial [10] and neurocognitive [11,12] functioning similar to that of healthy subjects, in contrast, at the other end, with individuals who show a severe neurocognitive and functional impairment $[13,14]$.

As with BD, schizophrenia is a heterogeneous disorder which is also associated with high levels of neurocognitive and psychosocial impairment $[15,16]$. Moreover, it has been shown that BD and SZ share a substantial genetic risk, although there are also specific loci which are responsible for the phenotypic differences existing between both disorders [17]. Thus, the comparison of the neurocognitive and psychosocial functioning of patients with BD and SZ has not only clinical interest, but it is 
also of relevance in order to establish possible shared pathophysiological mechanisms.

It has been suggested that patients with BD with a history of psychotic symptoms (BD-P) could constitute a phenotypically homogeneous subtype characterized by a greater neurocognitive $[18,19]$ and functional $[20,21]$ impairment. Thus, some authors have suggested that BD-P could occupy an intermediate position between patients with schizophrenia (SZ) and patients with BD without a history of psychotic symptoms (BD-NP) [22,23]. However, a number of cross sectional studies [24-26], including those from our group [27-29], did not find any relevant difference of neurocognitive and functional performance between BD-P and BD-NP, showing only subtle differences, and circumscribed to certain neurocognitive domains, such as working memory [28], or to specific areas of functioning, such as financial issues or occupational functioning $[29,30]$.

To the date, most longitudinal studies have shown stability in the neurocognitive deficit of patients with BD [2,31-33]. Similarly, no progression of the functional deficit has been found [34,35]. Nevertheless, it cannot be dismissed the possibility of the existence of a subset of patients with BD showing a progression of neurocognitive or functional outcome [36,37]. In this regard, both cross-sectional and longitudinal studies have pointed out that the neurocognitive and functional trajectory may be mediated by the clinical course $[38,39]$. Thus, some studies have suggested the possibility that clinical characteristics, such as the presence of episodes with psychotic symptoms, have a deleterious effect on the neurocognitive or functional trajectory of this disorder, although there are discrepant results [20,28,29,40-43]. Long-term studies are necessary to confirm these results [44].

To further extend our previous studies, we have carried out a 5year follow-up study aimed at comparing: 1) the course of neurocognitive dysfunction in a group of bipolar patients with and without a history of psychotic symptoms and a group of patients with schizophrenia, in relation to a healthy control group, 2) the course of functional impairment in these three groups of patients in relation to the control group, and 3) the severity of neurocognitive and functional impairment of the three groups of patients. We hypothesized that neurocognitive and functional deficits have a stable course both in patients with bipolar disorder and in patients with schizophrenia. Likewise, considering our previous results, we also hypothesized that there are no significant neurocognitive or functional differences between the two groups of patients with bipolar disorder.

\section{Material and methods}

\subsection{Sample}

Participants were enrolled in the Cuenca Bipolar Disorder Follow-up Study, a prospective study carried out at the Department of Psychiatry of the Hospital Virgen de la Luz de Cuenca (Castilla-La Mancha, Spain). The study was approved by the Clinical Research Ethics Committee of the Cuenca Health Area. All subjects gave written informed consent, after a complete description of the procedures and prior to enrolment in the study.

The description of the sample was extensively collected in previous cross-sectional studies [28,29]. Briefly, 100 patients with bipolar disorder type I, 50 of them who had a lifetime history of psychosis and 50 who had never presented psychotic symptoms, and 50 patients with schizophrenia were included in the study. All patients met DSM-IV criteria for schizophrenia or bipolar disorder. The diagnoses were confirmed by the Structured Clinical Interview for DSM-IV (SCID-I). The presence or absence of a history of psychotic symptoms (delusions and/or hallucinations) was established using the SCID-I, after carrying out a comprehensive review of the psychiatric chart. Variables of the course of illness such as the number of episodes and number of hospitalizations recorded both at baseline, and during the five-year follow up, were also ascertained reviewing the medical chart.

Patients with BD were euthymic for at least three months prior to the assessments. Euthymia was defined according to the following criteria [45]: a score fewer than 7 points on the Hamilton Depression Rating Scale (HAM-D) and a score fewer than 6 points on the Young Mania Rating Scale (YMRS). In addition, these scores remained below that threshold in three consecutive monthly evaluations. Moreover, all patients with SZ were clinically stabilized, at least during the three months before the assessments, according to criteria used in previous studies [27-29].

The exclusion criteria were as follows: (i) comorbid medical disease which can cause neurocognitive impairment, (ii) neuropsychiatric illness, other than SZ or BD, associated with cognitive impairment, (iii) drug abuse or dependence in the last 24 months, except nicotine and caffeine, (iv) a history of electroconvulsive therapy, (v) a history of brain trauma with loss of consciousness, (vi) less than six years of education, (vii) current IQ score lower than 70, (viii) refusal to sign written informed consent.

Fifty-one healthy controls (HC), matched with patients for age, gender and years of education, were enrolled in the study. In order to rule out the presence of psychiatric disorders, all control subjects were assessed with the SCID-I. The control group met the same exclusion criteria as patients, adding as an additional criterion for exclusion in this group the presence of some firstdegree relative diagnosed with a severe mental disorder (bipolar disorder or psychosis). The control subjects were living in the same catchment area and had the same ethnic origin as patients.

Both patients and HC were clinical, neuropsychologically and functionally assessed at two time points: at the beginning of the study (T1), and after a five-year follow-up (T2). The same inclusion and exclusion criteria considered at T1 were used at T2, thus euthymia and stability, as previously defined, were present in all subjects.

\subsection{Clinical and functional assessment}

Clinical assessments were carried out using the Spanish version of the following scales: The Positive and Negative Symptoms Scale (PANSS) [46], the Hamilton Depression Rating Scale [47], and the Young Mania Rating Scale [48].

Functional performance was evaluated using the Functioning Assessment Short Test (FAST) [49], and the function dimension of the split version of the Global Assessment of Functioning scale (GAF-F) [50]. The GAF-F is a 100-point scale reflecting functioning which scores from 1 , representing the most severe impairment, to 100 , representing the least impaired individual. The FAST is a scale which was primarily developed to assess psychosocial functioning in bipolar disorder [49], but that was later validated for schizophrenia [51,52]. This 24-items scale evaluates impairment during the last two weeks in six specific areas of functioning: autonomy, occupational functioning, cognitive functioning, financial issues, interpersonal relationships and leisure time. Each item is scored on a $0-3$-point scale (0: no difficulty, 1 : mild difficulty, 2 : moderate difficulty, 3: severe difficulty; total score ranges from 0 to 72 points), thus higher scores indicate poorer performance.

For every patient, the information was obtained from the participant, and at least, from a second source of reliable information (family, caregiver or case-management).

\subsection{Neuropsychological assessment}

Six neurocognitive domains, which correspond mostly to the domains included in the MATRICS Consensus Cognitive Battery [53], were evaluated through 12 neurocognitive measures, as 
follows: 1) Speed of Processing. Trail Making Test-Part A (TMT-A), WAIS-III digit-symbol coding subtest, Category Fluency Test (animal naming); 2) Working Memory. WAIS-III digit span backward subtest, letter-number sequencing subtest of the Wechsler Memory Scale (WMS-III); 3) Attention/vigilance. Degraded Stimulus Continuous Performance Test (DS-CPT); 4) Verbal Learning and Memory. California Verbal Learning Test (CVLT); 5) Visual memory. Rey-Osterrieth Complex Figure Test (ROCFT); 6) Executive Functions. Wisconsin Card Sorting Test (WCST), Stroop Test interference, Trail Making Test-Part B (TMT-B), FAS test. The value of the Cronbach's alpha for the six neurocognitive domains was 0.721 for speed of processing, 0.586 for working memory, 0.828 for executive function, 0.937 for visual memory, 0.826 for verbal memory, and 0.854 for attention. Additionally, premorbid IQ was determined using the vocabulary subtest of the Wechsler Adult Intelligence Scale WAIS-III.

\subsection{Statistical analysis}

The IBM SPSS Statistics 24 (Chicago IL, USA) for Windows was used for data analysis. Firstly, we examined if the study variables were normally distributed, and transformations were performed when necessary. For demographic and symptomatic variables, group differences were evaluated using one-way analysis of variance (ANOVA) (continuous variables) or chi-square test (categorical variables). For the analysis of neurocognitive performance, each measure in both T1 and T2 was first converted into zscores based on controls's scores at T1. Data were also transformed so that higher scores always indicated better performance. The $\mathrm{z}$ scores that were obtained approached a normal distribution with a mean of 0 and a standard deviation of 1 . Scores of neurocognitive domains composed of more than one measure were obtained by averaging the z-scores of all measures in the domain. Also, a neurocognitive composite index $(\mathrm{NCI})$ was calculated by averaging the scores obtained in each cognitive domain.

To assess longitudinal neurocognitive and functional performance, repeated measures ANOVA was used for each neurocognitive and functional measure including the normalized $\mathrm{T} 1$ and $\mathrm{T} 2$ values based on controls $\mathrm{T} 1$ mean and standard deviation, in order to adjust each change comparison for the baseline values. Effects of group, time, and group-by-time interactions were examined. Bonferroni test was used for post hoc analyses. Scores on affective symptom scales (HAM-D and YMRS) were used as covariates when significant differences between groups were found.

\section{Results}

One-hundred and fifty patients (50 BD-P, 50 BD-NP, $50 \mathrm{SZ}$ ), and 51 $\mathrm{HC}$ were initially included in this study. At the end of the 5-year follow-up, 37 patients (6 BP-P, 16 BD-NP, 15 SZ) were lost. Of these, 2 patients died (committed suicide), 12 did not meet the inclusion criteria at T2, and 23 patients refused to be evaluated at T2. Similarly, $15 \mathrm{HC}$ refused to be assessed at $\mathrm{T} 2$. We found no significant differences in demographic, clinical, cognitive, functional, or treatment variables at $\mathrm{T} 1$ between subjects who completed and those who did not complete the follow-up for any reason.

Table 1

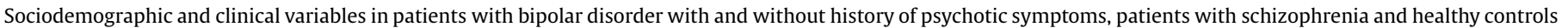
at baseline and after a five-year follow-up.

\begin{tabular}{|c|c|c|c|c|c|c|}
\hline & $\begin{array}{l}\mathrm{HC} \\
\mathrm{N}=36\end{array}$ & $\begin{array}{l}\text { BD-NP } \\
\mathrm{N}=34\end{array}$ & $\begin{array}{l}\text { BD-P } \\
\mathrm{N}=44\end{array}$ & $\begin{array}{l}\mathrm{SZ} \\
\mathrm{N}=35\end{array}$ & Significance & \\
\hline Females No (\%) & $17(47.2)$ & $20(58.8)$ & $23(52.3)$ & $17(48.6)$ & 0.772 & \\
\hline Age (years) & $42.6(13.2)$ & $42.1(12.2)$ & $40.6(10.7)$ & $39.1(9.2)$ & 0.571 & \\
\hline Education (years) & $12.7(3.0)$ & $12.7(4.0)$ & $12.3(3.4)$ & $12.1(2.9)$ & 0.858 & \\
\hline Age at onset (years) & - & $28.3(9.1)$ & $24.6(9.1)$ & $23.1(5.6)$ & 0.028 & $\mathrm{SZ}<\mathrm{BD}-\mathrm{NP}$ \\
\hline Duration of illness (years) & - & $13.9(10.1)$ & $16.3(10.7)$ & $16.0(7.5)$ & 0.532 & \\
\hline HAM-D score at $\mathrm{T} 1$ & $1.3(1.1)$ & $2.8(2.1)$ & $2.6(2.2)$ & $3.7(2.1)$ & 0.000 & BD-NP, BD-P, SZ > HC \\
\hline YMRS score at T1 & $0.3(0.7)$ & $0.3(1.1)$ & $0.4(0.1)$ & $1.2(2.05)$ & 0.009 & $\mathrm{SZ}>\mathrm{BD}-\mathrm{P}, \mathrm{BD}-\mathrm{NP}, \mathrm{HC}$ \\
\hline HAM-D score at T2 & $1.6(1.4)$ & $2.8(2.4)$ & $3.0(2.8)$ & $3.9(2.5)$ & 0.002 & $\mathrm{SZ}>\mathrm{HC}$ \\
\hline YMRS score at T2 & $0.7(0.9)$ & $1.5(2.3)$ & $0.7(1.3)$ & $1.3(2.9)$ & 0.182 & \\
\hline PANSS-T score at T1 & & & & $53.1(13.6)$ & & \\
\hline PANSS-P score at T1 & & & & $11.1(3.5)$ & & \\
\hline PANSS-N score at T1 & & & & $16.2(7.3)$ & & \\
\hline PANSS-PG score at T1 & & & & $25.7(6.5)$ & & \\
\hline PANSS-T score at T2 & & & & $53.7(13.3)$ & & \\
\hline PANSS-P score at T2 & & & & $11.5(3.3)$ & & \\
\hline PANSS-N score at T2 & & & & $17.2(7.2)$ & & \\
\hline PANSS-PG score at T2 & & & & $25.1(5.8)$ & & \\
\hline Premorbid IQ & $100.0(10.0)$ & $98.3(15.0)$ & $92.8(12.8)$ & $91.2(12.5)$ & 0.008 & $\mathrm{HC}>\mathrm{BD}-\mathrm{P}, \mathrm{SZ}$ \\
\hline No. hospitalizations at $\mathrm{T} 1$ & - & $2.5(4.8)$ & $4.11(4.8)$ & $3.31(3.6)$ & 0.272 & \\
\hline No. hospitalizations for depression at $\mathrm{T} 1$ & - & $1.29(4.8)$ & $1.14(2.5)$ & - & $0.852^{a}$ & \\
\hline No. hospitalizations for mania at $\mathrm{T} 1$ & - & $1.15(1.5)$ & $2.98(4.0)$ & - & $0.007^{\mathrm{a}}$ & $\mathrm{BD}-\mathrm{P}>\mathrm{BD}-\mathrm{NP}$ \\
\hline No. of depressive episodes at $\mathrm{T} 1$ & - & $10.59(10.6)$ & $7.73(7.2)$ & - & $0.182^{\mathrm{a}}$ & \\
\hline No. of manic/hypomanic episodes at $\mathrm{T} 1$ & - & $3.65(4.8)$ & $5.95(5.3)$ & - & $0.050^{\mathrm{a}}$ & \\
\hline No. total of episodes at $\mathrm{T} 1$ & - & $19.00(17.7)$ & $15.84(15.4)$ & - & $0.405^{\mathrm{a}}$ & \\
\hline \multicolumn{7}{|l|}{ Course of illness during the follow up period. } \\
\hline No. of hospitalizations & - & $0.61(1.1)$ & $0.89(1.4)$ & $1.09(1.8)$ & 0.409 & \\
\hline No. of hospitalizations for depression & - & $0.24(0.75)$ & $0.32(1.1)$ & $0.09(0.38)$ & $0.726^{\mathrm{a}}$ & \\
\hline No. of hospitalizations for mania & - & $0.33(0.6)$ & $0.52(1.0)$ & - & $0.337^{\mathrm{a}}$ & \\
\hline No. of hospitalizations for psychosis (non-affective) & - & - & - & $0.97(1.7)$ & & \\
\hline No. of depressive episodes & & $1.88(3.3)$ & $1.82(2.7)$ & $0.21(0.6)$ & $0.929^{\mathrm{a}}$ & \\
\hline No. of manic/hypomanic episodes & & $1.64(2.8)$ & $1.57(2.1)$ & - & $0.905^{\mathrm{a}}$ & \\
\hline No. total of episodes & & $3.48(5.9)$ & $3.34(4.3)$ & & $0.902^{\mathrm{a}}$ & \\
\hline
\end{tabular}

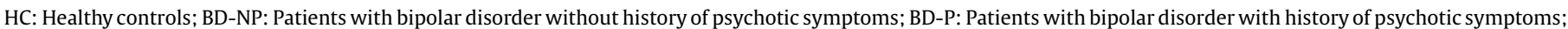

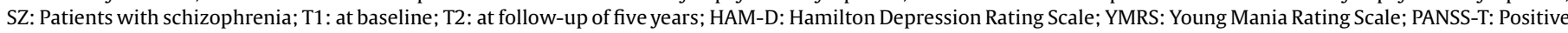

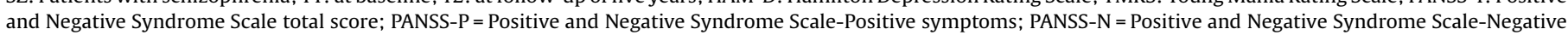
symptoms; PANSS-PG = Positive and Negative Syndrome Scale-General psychopathology; IQ: Intelligence quotient.

${ }^{a}$ Only BD-P and BD-NP were analyzed. 
The time elapsed between the first and second assessments was 63.8 months [standard deviation $(\mathrm{SD})=5.2$ months; range: $55-78$ months] for patients and 62.4 months ( $\mathrm{SD}=8.1$ months; range: 51 76 months) for the control group ( $\mathrm{p}=0.331 ; \mathrm{F}=0.953)$.

Table 1 shows sociodemographic and clinical characteristics of patients and control subjects which completed the follow-up. There were no significant differences between the four groups at baseline either for age, years of education or for gender distribution. HC had higher premorbid IQ than patients with BD-P and SZ. The three groups of patients scored higher on the HAM-D than HC at T1, although, at the end of the follow-up period, differences were only found between HC and SZ. Moreover, on the YMRS, patients with SZ scored higher than patients with BD and HC at T1.

Comparing the three groups of patients, no differences were found in years of evolution of illness. Nevertheless, patients with SZ had an earlier onset than BD-NP. When we compared the course of illness between BD-P and BD-NP, at baseline, patients did not differ in number of hospitalizations, number of hospitalizations for depression, or number of episodes (total, depressive or manic/ hypomanic), differing however on number of hospitalizations for mania, which were significantly higher in BD-P than in BD-NP. The characteristics of pharmacological treatment are displayed in Table 2. A greater number of patients with $\mathrm{BD}-\mathrm{P}\left(\mathrm{Chi}^{2}=11.857\right.$; df: $1 ; \mathrm{p}<0.001)$, but no of patients with BD-NP $\left(\mathrm{Chi}^{2}=2.893\right.$; df: 1 ; $\mathrm{p}<0.089$ ) received antipsychotic treatment at $\mathrm{T} 2$ in relation to $\mathrm{T} 1$.

\subsection{Neurocognitive function}

Table 3 shows the $z$-scores and results of the repeated-measures ANOVA for each neurocognitive measure (See Fig. 1 and Supplemental Fig. 1). We found an effect of time on some cognitive measures included in the domains executive function and attention (worse performance both at $\mathrm{T} 2$ than at $\mathrm{T} 1$ in the domain of attention, and at T1 than at T2 in executive function). However, we did not find a group $\mathrm{x}$ time interaction for any cognitive measure.

We found an effect of group for each neurocognitive measure, except for recognition (verbal memory domain) and verbal fluency.
The results of the post-hoc tests (Bonferroni test) are shown in Table 4. For the $\mathrm{NCI}, \mathrm{HC}$ had higher scores than patients. Nevertheless, no significant differences were found between the three groups of patients. Similar results were found for the domains speed processing, verbal memory, visual memory and executive function. For the domains attention and working memory, HC scored higher than BD-P and SZ, but not than BDNP. Additionally, in the working memory domain, there were significant differences between BD-NP and SZ. We only found differences between BD-P and BD-NP in one measure of the domain working memory (letter and number).

\subsection{Psychosocial functioning}

Repeated-measures ANOVA showed group $\mathrm{x}$ time interaction for no functional measure (Table 5), although there was an effect of time on the GAF-F (worse performance at T2 than at T1).

We found an effect of group for each functional measure (Table 5). The results of the post-hoc tests are shown in Table 4. There were differences between HC and BD-NP in no functional measure, except for the subscale of the FAST leisure time. However, HC had better performance than BD-P in the two measures of overall functioning and in the subscales occupational functioning, interpersonal relationships and leisure time. Additionally, HC had better performance than SZ in all functional measures.

BD-NP had better performance than BD-P in the two measures of overall functioning and the subscales occupational functioning and interpersonal relationships. Furthermore, BD-NP had better performance than SZ in all functional measures, except for financial issues. BD-P had better performance than SZ in the measures of overall functioning (GAF-F and FAST-total score), and the subscales autonomy, cognitive functioning, and interpersonal relationships (See Fig. 1 and Supplemental Fig. 2).

\section{Discussion}

In the present study, we compared the neurocognitive and functional course of euthymic patients with bipolar disorder, with

Table 2

Pharmacological treatment in patients with bipolar disorder and patients with schizophrenia.

\begin{tabular}{|c|c|c|c|c|c|c|c|c|}
\hline & $\mathrm{T} 1$ & & & & $\mathrm{~T} 2$ & & & \\
\hline & BD-NP & BD-P & SZ & $\mathbf{p}$ & BD-NP & BD-P & SZ & $\mathbf{p}$ \\
\hline & $N=34$ & $N=44$ & $\mathrm{~N}=35$ & & $N=34$ & $N=44$ & $\mathrm{~N}=35$ & \\
\hline & n (\%) & n (\%) & n (\%) & & n (\%) & n (\%) & n (\%) & \\
\hline Lithium (monotherapy) & $13(38.2 \%)$ & $13(29.5 \%)$ & - & $0.419^{1}$ & $7(20.6 \%)$ & $6(13.6 \%)$ & - & $0.380^{1}$ \\
\hline Anticonvulsant (monotherapy) & $12(35.3 \%)$ & $9(20.5 \%)$ & - & $0.143^{1}$ & $9(26.5 \%)$ & $7(15.9 \%)$ & $1(2.9 \%)$ & $0.224^{1, *}$ \\
\hline Lithium + Anticonvulsant & $5(14.7 \%)$ & $11(25 \%)$ & - & $0.264^{1}$ & $5(14.7 \%)$ & $4(9.1 \%)$ & - & $0.413^{1}$ \\
\hline Lithium + SGA & $3(8.8 \%)$ & $5(11.4 \%)$ & - & $0.134^{1}$ & $7(20.6 \%)$ & $11(25 \%)$ & - & $0.698^{1}$ \\
\hline Lithium + Anticonvulsant + SGA & $0(0 \%)$ & $3(6.8 \%)$ & - & $0.120^{1}$ & $1(2.9 \%)$ & $4(9.1 \%)$ & - & $0.285^{1}$ \\
\hline Anticonvulsant + SGA & $2(5.9 \%)$ & $2(4.5 \%)$ & - & $0.790^{1}$ & $2(5.9 \%)$ & $7(15.9 \%)$ & - & $0.183^{1}$ \\
\hline SGA without MS & $0(0 \%)$ & $2(4.5 \%)$ & $35(100 \%)$ & & $1(2.9 \%)$ & $4(9.1 \%)$ & $34(97.1 \%)$ & $0.285^{1, *}$ \\
\hline SGA (with or without MS) & $5(14.7 \%)$ & $11(25 \%)$ & $35(100 \%)$ & $0.264^{1, *}$ & $11(32.4)$ & $27(61.4 \%)$ & $35(100 \%)$ & $0.015^{1, *}$ \\
\hline Antidepressants & $9(26.5 \%)$ & $10(22.7 \%)$ & $6(17.1 \%)$ & $0.703^{1, *}$ & $8(23.5 \%)$ & $10(22.7 \%)$ & $5(14.3 \%)$ & $0.876^{1, *}$ \\
\hline \multirow[t]{2}{*}{ Benzodiazepines } & $7(20.6 \%)$ & $9(20.5 \%)$ & $15(42.9 \%)$ & $0.988^{1, *}$ & $13(38.2 \%)$ & $16(36.4 \%)$ & $15(42.9 \%)$ & $0.786^{1, *}$ \\
\hline & $\begin{array}{l}\text { BD-NP } \\
\text { mean (SD) }\end{array}$ & $\begin{array}{l}\text { BD-P } \\
\text { mean (SD) }\end{array}$ & $\begin{array}{l}\text { SZ } \\
\text { mean (SD) }\end{array}$ & $\mathbf{p}$ & $\begin{array}{l}\text { BD-NP } \\
\text { mean (SD }\end{array}$ & $\begin{array}{l}\text { BD-P } \\
\text { mean (SD) }\end{array}$ & $\begin{array}{l}\text { SZ } \\
\text { mean (SD) }\end{array}$ & $\mathbf{p}$ \\
\hline Number of MS & $1.09(0.29)$ & $1.23(0.52)$ & - & $0.133^{1}$ & $1.12(0.49)$ & $1.07(0.55)$ & - & $0.678^{1}$ \\
\hline Total number of psychotropic drugs & $1.71(0.87)$ & $2.02(0.88)$ & $1.83(0.95)$ & $0.184^{2}$ & $2.18(1.1)$ & $2.36(1.08)$ & $1.94(0.90)$ & $0.218^{2}$ \\
\hline Lithium levels (mEq / L) & $0.68(0.11)$ & $0.71(0.19)$ & - & $0.538^{3}$ & $0.65(0.09)$ & $0.65(0.12)$ & - & $0.988^{3}$ \\
\hline Dosage of antipsychotic (E-CPZ) & $205.00(125.5)$ & $226.64(150.0)$ & $452.1(290.9)$ & $0.784^{3}$ & $268.2(180.3)$ & $318.9(247.4)$ & $547.9(363.5)$ & $0.541^{3}$ \\
\hline
\end{tabular}

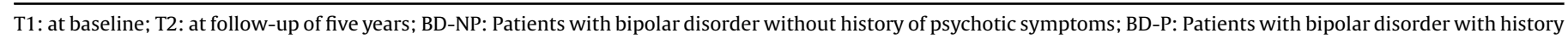
of psychotic symptoms; SZ: Patients with schizophrenia; SGA: Second-generation antipsychotics; MS: Mood stabilizers; E-CPZ: mg equivalents of Chlorpromazine.

Comparisons only between BD-P and BD-NP.

$1 \mathrm{Chi}^{2}$.

2 Mann-Whitney U test.

${ }^{3}$ Kruskal Wallis Test. 
Table 3

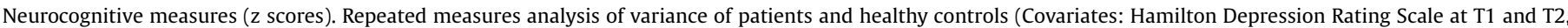
Young Mania rating scale at T1).

\begin{tabular}{|c|c|c|c|c|c|c|c|c|c|c|c|}
\hline & \multicolumn{4}{|l|}{ T1 } & \multicolumn{4}{|l|}{$\mathrm{T} 2$} & \multirow{2}{*}{$\begin{array}{l}\text { Group effect } \\
(G)\end{array}$} & \multirow{2}{*}{$\begin{array}{l}\text { Time effect } \\
(\mathrm{T})\end{array}$} & \multirow{2}{*}{$\begin{array}{l}\mathrm{G} \times \mathrm{T} \\
\text { Interaction }\end{array}$} \\
\hline & $\begin{array}{l}\mathrm{HC} \\
\mathrm{N}=36\end{array}$ & $\begin{array}{l}\text { BD-NP } \\
N=34\end{array}$ & $\begin{array}{l}\mathrm{BD}-\mathrm{P} \\
\mathrm{N}=44\end{array}$ & $\begin{array}{l}\mathrm{SZ} \\
\mathrm{N}=35\end{array}$ & $\begin{array}{l}\mathrm{HC} \\
\mathrm{N}=36\end{array}$ & $\begin{array}{l}\text { BD-NP } \\
\mathrm{N}=34\end{array}$ & $\begin{array}{l}\mathrm{BD}-\mathrm{P} \\
\mathrm{N}=44\end{array}$ & $\begin{array}{l}\mathrm{SZ} \\
\mathrm{N}=35\end{array}$ & & & \\
\hline $\mathrm{NCI}$ & $\begin{array}{l}0.00 \\
(0.5)\end{array}$ & $\begin{array}{l}-0.70 \\
(0.7)\end{array}$ & $\begin{array}{l}-0.89 \\
(0.7)\end{array}$ & $\begin{array}{l}-1.16 \\
(0.8)\end{array}$ & $\begin{array}{l}-0.10 \\
(0.6)\end{array}$ & $\begin{array}{l}-0.92 \\
(0.9)\end{array}$ & $\begin{array}{l}-1.02 \\
(0.9)\end{array}$ & $\begin{array}{l}-1.29 \\
(0.8)\end{array}$ & $0.000^{1}$ & 0.326 & 0.669 \\
\hline Speed processing index & $\begin{array}{l}0.00 \\
(0.7)\end{array}$ & $\begin{array}{l}-0.60 \\
(0.8)\end{array}$ & $\begin{array}{l}-0.82 \\
(0.9)\end{array}$ & $\begin{array}{l}-0.88 \\
(0.8)\end{array}$ & $0.06(0.7)$ & $\begin{array}{l}-0.69 \\
(1.0)\end{array}$ & $\begin{array}{l}-0.87 \\
(1.0)\end{array}$ & $\begin{array}{l}-1.08 \\
(0.9)\end{array}$ & $0.000^{1}$ & 0.385 & 0.307 \\
\hline TMT-A & $\begin{array}{l}0.00 \\
(1.0)\end{array}$ & $\begin{array}{l}-0.68 \\
(1.4)\end{array}$ & $\begin{array}{l}-0.88 \\
(1.5)\end{array}$ & $\begin{array}{l}-0.82 \\
(1.3)\end{array}$ & $0.15(0.8)$ & $\begin{array}{l}-0.60 \\
(1.5)\end{array}$ & $\begin{array}{l}-0.67 \\
(1.6)\end{array}$ & $\begin{array}{l}-0.91 \\
(1.5)\end{array}$ & $0.018^{2}$ & 0.894 & 0.304 \\
\hline Digit-symbol coding & $\begin{array}{l}0.00 \\
(1.0)\end{array}$ & $\begin{array}{l}-0.56 \\
(0.6)\end{array}$ & $\begin{array}{l}-0.66 \\
(0.7)\end{array}$ & $\begin{array}{l}-0.74 \\
(0.6)\end{array}$ & $0.20(1.0)$ & $\begin{array}{l}-0.72 \\
(0.8)\end{array}$ & $\begin{array}{l}-0.75 \\
(0.9)\end{array}$ & $\begin{array}{l}-1.09 \\
(1.0)\end{array}$ & $0.000^{1}$ & 0.292 & 0.071 \\
\hline Verbal fluency (categ) & $\begin{array}{l}0.00 \\
(1.0)\end{array}$ & $\begin{array}{l}-0.56 \\
(1.2)\end{array}$ & $\begin{array}{l}-0.93 \\
(1.2)\end{array}$ & $\begin{array}{l}-1.08 \\
(1.1)\end{array}$ & $\begin{array}{l}-0.17 \\
(1.0)\end{array}$ & $\begin{array}{l}-0.66 \\
(1.1)\end{array}$ & $\begin{array}{l}-1.24 \\
(1.0)\end{array}$ & $\begin{array}{l}-1.25 \\
(1.2)\end{array}$ & $0.000^{2}$ & 0.506 & 0.762 \\
\hline Working memory index & $\begin{array}{l}0.00 \\
(0.8)\end{array}$ & $\begin{array}{l}-0.10 \\
(1.2)\end{array}$ & $\begin{array}{l}-0.81 \\
(0.9)\end{array}$ & $\begin{array}{l}-1.13 \\
(1.1)\end{array}$ & $\begin{array}{l}-0.04 \\
(1.0)\end{array}$ & $\begin{array}{l}-0.41 \\
(1.2)\end{array}$ & $\begin{array}{l}-0.79 \\
(1.0)\end{array}$ & $\begin{array}{l}-1.01 \\
(1.2)\end{array}$ & $0.000^{4}$ & 0.712 & 0.160 \\
\hline Digit span backward & $\begin{array}{l}0.00 \\
(1.0)\end{array}$ & $\begin{array}{l}-0.14 \\
(1.5)\end{array}$ & $\begin{array}{l}-0.73 \\
(1.4)\end{array}$ & $\begin{array}{l}-1.02 \\
(1.4)\end{array}$ & $0.15(1.2)$ & $\begin{array}{l}-0.53 \\
(1.6)\end{array}$ & $\begin{array}{l}-0.73 \\
(1.4)\end{array}$ & $\begin{array}{l}-0.89 \\
(1.5)\end{array}$ & $0.010^{2}$ & 0.755 & 0.275 \\
\hline Letter-number & $\begin{array}{l}0.00 \\
(1.0)\end{array}$ & $\begin{array}{l}-0.06 \\
(1.0)\end{array}$ & $\begin{array}{l}-0.88 \\
(0.7)\end{array}$ & $\begin{array}{l}-1.23 \\
(1.1)\end{array}$ & $\begin{array}{l}-0.19 \\
(1.1)\end{array}$ & $\begin{array}{l}-0.29 \\
(1.2)\end{array}$ & $\begin{array}{l}-0.84 \\
(1.0)\end{array}$ & $\begin{array}{l}-1.14 \\
(1.1)\end{array}$ & $0.000^{3}$ & 0.194 & 0.393 \\
\hline Verbal memory index & $\begin{array}{l}0.00 \\
(0.9)\end{array}$ & $\begin{array}{l}-0.67 \\
(0.8)\end{array}$ & $\begin{array}{l}-0.77 \\
(0.8)\end{array}$ & $\begin{array}{l}-0.90 \\
(1.0)\end{array}$ & $0.03(1.0)$ & $\begin{array}{l}-0.77 \\
(0.9)\end{array}$ & $\begin{array}{l}-0.69 \\
(0.8)\end{array}$ & $\begin{array}{l}-0.94 \\
(1.0)\end{array}$ & $0.004^{1}$ & 0.549 & 0.611 \\
\hline Learning & $\begin{array}{l}0.00 \\
(1.0)\end{array}$ & $\begin{array}{l}-0.73 \\
(0.9)\end{array}$ & $\begin{array}{l}-0.91 \\
(0.9)\end{array}$ & $\begin{array}{l}-1.10 \\
(1.0)\end{array}$ & $\begin{array}{l}-0.11 \\
(1.0)\end{array}$ & $\begin{array}{l}-1.08 \\
(1.2)\end{array}$ & $\begin{array}{l}-0.97 \\
(0.9)\end{array}$ & $\begin{array}{l}-1.39 \\
(1.1)\end{array}$ & $0.001^{1}$ & 0.313 & 0.390 \\
\hline Immediate free recall & $\begin{array}{l}0.00 \\
(1.0)\end{array}$ & $\begin{array}{l}-0.75 \\
(0.9)\end{array}$ & $\begin{array}{l}-0.86 \\
(0.9)\end{array}$ & $\begin{array}{l}-0.98 \\
(1.1)\end{array}$ & $0.07(1.0)$ & $\begin{array}{l}-0.76 \\
(1.0)\end{array}$ & $\begin{array}{l}-0.76 \\
(1.0)\end{array}$ & $\begin{array}{l}-1.11 \\
(1.2)\end{array}$ & $0.001^{1}$ & 0.839 & 0.734 \\
\hline Delayed free recall & $\begin{array}{l}0.00 \\
(1.0)\end{array}$ & $\begin{array}{l}-0.83 \\
(1.1)\end{array}$ & $\begin{array}{l}-0.97 \\
(1.0)\end{array}$ & $\begin{array}{l}-1.08 \\
(1.2)\end{array}$ & $0.20(1.0)$ & $\begin{array}{l}-0.90 \\
(1.1)\end{array}$ & $\begin{array}{l}-0.69 \\
(0.9)\end{array}$ & $\begin{array}{l}-1.03 \\
(1.3)\end{array}$ & $0.001^{1}$ & 0.377 & 0.282 \\
\hline Recognition & $\begin{array}{l}0.00 \\
(1.0)\end{array}$ & $\begin{array}{l}-0.38 \\
(0.9)\end{array}$ & $\begin{array}{l}-0.31 \\
(1.0)\end{array}$ & $\begin{array}{l}-0.44 \\
(1.5)\end{array}$ & $\begin{array}{l}-0.04 \\
(1.1)\end{array}$ & $\begin{array}{l}-0.34 \\
(1.4)\end{array}$ & $\begin{array}{l}-0.33 \\
(1.3)\end{array}$ & $\begin{array}{l}-0.23 \\
(1.0)\end{array}$ & 0.737 & 0.204 & 0.940 \\
\hline Visual memory index & $\begin{array}{l}0.00 \\
(1.0)\end{array}$ & $\begin{array}{l}-1.22 \\
(1.4)\end{array}$ & $\begin{array}{l}-1.18 \\
(1.4)\end{array}$ & $\begin{array}{l}-1.63 \\
(1.3)\end{array}$ & $\begin{array}{l}-0.19 \\
(1.2)\end{array}$ & $\begin{array}{l}-1.59 \\
(1.5)\end{array}$ & $\begin{array}{l}-1.33 \\
(1.5)\end{array}$ & $\begin{array}{l}-1.61 \\
(1.8)\end{array}$ & $0.000^{1}$ & 0.940 & 0.492 \\
\hline ROCFT immediate recall & $\begin{array}{l}0.00 \\
(1.0)\end{array}$ & $\begin{array}{l}-1.26 \\
(1.5)\end{array}$ & $\begin{array}{l}-1.19 \\
(1.6)\end{array}$ & $\begin{array}{l}-1.78 \\
(1.5)\end{array}$ & $\begin{array}{l}-0.20 \\
(1.3)\end{array}$ & $\begin{array}{l}-1.68 \\
(1.6)\end{array}$ & $\begin{array}{l}-1.43 \\
(1.6)\end{array}$ & $\begin{array}{l}-1.75 \\
(1.9)\end{array}$ & $0.000^{1}$ & 0.797 & 0.486 \\
\hline ROCFT delayed recall & $\begin{array}{l}0.00 \\
(1.0)\end{array}$ & $\begin{array}{l}-1.19 \\
(1.4)\end{array}$ & $\begin{array}{l}-1.16 \\
(1.4)\end{array}$ & $\begin{array}{l}-1.49 \\
(1.3)\end{array}$ & $\begin{array}{l}-0.20 \\
(1.1)\end{array}$ & $\begin{array}{l}-1.49 \\
(1.5)\end{array}$ & $\begin{array}{l}-1.22 \\
(1.4)\end{array}$ & $\begin{array}{l}-1.47 \\
(1.8)\end{array}$ & $0.000^{1}$ & 0.888 & 0.546 \\
\hline $\begin{array}{l}\text { Executive function } \\
\text { index }\end{array}$ & $\begin{array}{l}0.00 \\
(0.7)\end{array}$ & $\begin{array}{l}-0.96 \\
(0.9)\end{array}$ & $\begin{array}{l}-1.00 \\
(1.2)\end{array}$ & $\begin{array}{l}-1.32 \\
(1.2)\end{array}$ & $0.03(0.7)$ & $\begin{array}{l}-0.70 \\
(1.1)\end{array}$ & $\begin{array}{l}-0.72 \\
(1.2)\end{array}$ & $\begin{array}{l}-1.17 \\
(1.1)\end{array}$ & $0.000^{1}$ & 0.007 & 0.275 \\
\hline WCST No. categories & $\begin{array}{l}0.00 \\
(1.0)\end{array}$ & $\begin{array}{l}-1.15 \\
(1.3)\end{array}$ & $\begin{array}{l}-0.89 \\
(1.4)\end{array}$ & $\begin{array}{l}-1.21 \\
(1.3)\end{array}$ & $0.22(0.8)$ & $\begin{array}{l}-0.46 \\
(1.2)\end{array}$ & $\begin{array}{l}-0.40 \\
(1.2)\end{array}$ & $\begin{array}{l}-1.05 \\
(1.4)\end{array}$ & $0.001^{1}$ & 0.001 & 0.256 \\
\hline $\begin{array}{l}\text { WCST perseverative } \\
\text { errors }\end{array}$ & $\begin{array}{l}0.00 \\
(1.0)\end{array}$ & $\begin{array}{l}-1.22 \\
(1.4)\end{array}$ & $\begin{array}{l}-1.21 \\
(1.4)\end{array}$ & $\begin{array}{l}-1.80 \\
(1.8)\end{array}$ & $0.06(0.8)$ & $\begin{array}{l}-0.96 \\
(1.7)\end{array}$ & $\begin{array}{l}-0.66 \\
(1.5)\end{array}$ & $\begin{array}{l}-1.37 \\
(1.6)\end{array}$ & $0.000^{1}$ & 0.000 & 0.126 \\
\hline TMT-B & $\begin{array}{l}0.00 \\
(1.0)\end{array}$ & $\begin{array}{l}-1.26 \\
(1.5)\end{array}$ & $\begin{array}{l}-1.30 \\
(1.8)\end{array}$ & $\begin{array}{l}-1.53 \\
(1.9)\end{array}$ & $\begin{array}{l}-0.03 \\
(1.0)\end{array}$ & $\begin{array}{l}-0.94 \\
(1.4)\end{array}$ & $\begin{array}{l}-1.05 \\
(1.9)\end{array}$ & $\begin{array}{l}-1.46 \\
(1.6)\end{array}$ & $0.001^{1}$ & 0.678 & 0.772 \\
\hline Verbal fluency (FAS) & $\begin{array}{l}0.00 \\
(1.0)\end{array}$ & $\begin{array}{l}-0.37 \\
(0.9)\end{array}$ & $\begin{array}{l}-0.45 \\
(0.9)\end{array}$ & $\begin{array}{l}-0.53 \\
(1.0)\end{array}$ & $\begin{array}{l}-0.04 \\
(0.9)\end{array}$ & $\begin{array}{l}-0.20 \\
(1.1)\end{array}$ & $\begin{array}{l}-0.46 \\
(1.2)\end{array}$ & $\begin{array}{l}-0.49 \\
(0.8)\end{array}$ & 0.080 & 0.253 & 0.715 \\
\hline Stroop & $\begin{array}{l}0.00 \\
(1.0)\end{array}$ & $\begin{array}{l}-0.79 \\
(1.5)\end{array}$ & $\begin{array}{l}-1.16 \\
(2.0)\end{array}$ & $\begin{array}{l}-1.51 \\
(2.3)\end{array}$ & $\begin{array}{l}-0.20 \\
(1.5)\end{array}$ & $\begin{array}{l}-0.95 \\
(1.8)\end{array}$ & $\begin{array}{l}-1.03 \\
(1.8)\end{array}$ & $\begin{array}{l}-1.47 \\
(1.9)\end{array}$ & $0.013^{5}$ & 0.784 & 0.778 \\
\hline Attention index & $\begin{array}{l}0.00 \\
(0.5)\end{array}$ & $\begin{array}{l}-0.40 \\
(0.8)\end{array}$ & $\begin{array}{l}-0.45 \\
(0.8)\end{array}$ & $\begin{array}{l}-0.52 \\
(0.8)\end{array}$ & $\begin{array}{l}-0.33 \\
(0.7)\end{array}$ & $\begin{array}{l}-0.79 \\
(1.0)\end{array}$ & $\begin{array}{l}-0.99 \\
(1.0)\end{array}$ & $\begin{array}{l}-1.08 \\
(1.1)\end{array}$ & $0.002^{2}$ & 0.041 & 0.721 \\
\hline CPT hits & $\begin{array}{l}0.00 \\
(1.0)\end{array}$ & $\begin{array}{l}-0.75 \\
(1.5)\end{array}$ & $\begin{array}{l}-0.83 \\
(1.4)\end{array}$ & $\begin{array}{l}-0.92 \\
(1.4)\end{array}$ & $\begin{array}{l}-0.59 \\
(1.3)\end{array}$ & $\begin{array}{l}-1.45 \\
(1.8)\end{array}$ & $\begin{array}{l}-1.82 \\
(1.8)\end{array}$ & $\begin{array}{l}-1.97 \\
(2.0)\end{array}$ & $0.011^{2}$ & 0.021 & 0.815 \\
\hline CPT sensitivity $A^{\prime}$ & $\begin{array}{l}0.00 \\
(1.0)\end{array}$ & $\begin{array}{l}-0.55 \\
(1.5)\end{array}$ & $\begin{array}{l}-0.73 \\
(1.7)\end{array}$ & $\begin{array}{l}-1.28 \\
(2.6)\end{array}$ & $\begin{array}{l}-0.42 \\
(1.2)\end{array}$ & $\begin{array}{l}-1.22 \\
(1.9)\end{array}$ & $\begin{array}{l}-1.64 \\
(2.1)\end{array}$ & $\begin{array}{l}-1.84 \\
(2.3)\end{array}$ & $0.010^{5}$ & 0.131 & 0.617 \\
\hline
\end{tabular}

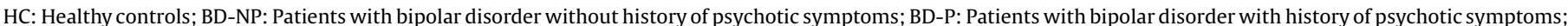

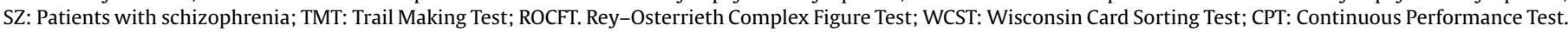

$1 \mathrm{HC}>\mathrm{BD}-\mathrm{NP}, \mathrm{BD}-\mathrm{P}, \mathrm{SZ}$.

2 HC > BD-P, SZ.

${ }^{3}$ HC, BD-NP $>$ BD-P, SZ.

${ }^{4} \mathrm{HC}>\mathrm{BD}-\mathrm{P}, \mathrm{SZ} ; \mathrm{BD}-\mathrm{NP}>\mathrm{SZ}$.

${ }^{5} \mathrm{HC}>\mathrm{SZ}$.

and without a lifetime history of psychosis, a group of stabilized patients with schizophrenia, and a group of healthy controls, during a five-year follow-up. Three main findings should be highlighted.

First, we did not find evidence of progression either in the neurocognitive or in the psychosocial impairment in any of the three groups of patients in relation to HC. The stability found in the neurocognitive impairment is in accordance with the results obtained in most longitudinal studies which have examined the course of neurocognitive function both in patients with $\mathrm{BD}$
[31,54-56], and patients with SZ [57-59]. Likewise, in accordance with our results, other authors could not establish a progressive course of the psychosocial impairment in patients with BD [35], or in patients with SZ $[60,61]$. In this regard, although it cannot be dismissed the possibility of a subset of patients with BD with a progressive course [39], our results suggest that the presence of a history of psychotic symptoms is not associated with a progression of the neurocognitive or psychosocial impairment.

Second, in line with the results obtained in our cross-sectional study [28], BD-P and BD-NP have a neurocognitive profile of similar 
A

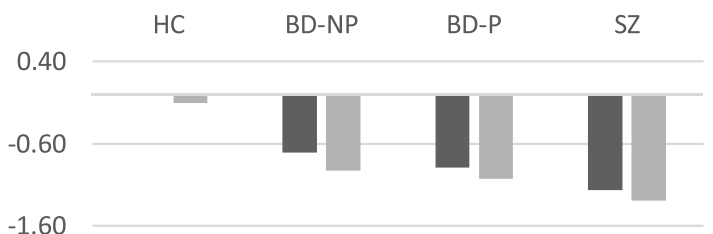

$\square \mathrm{NCl}$ T1 $\mathrm{NCl} \mathrm{T2}$

B

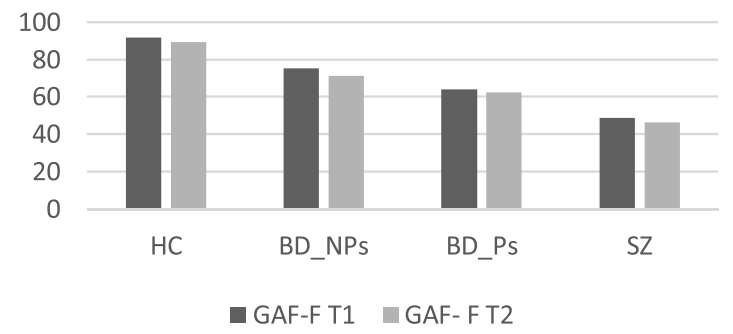

C

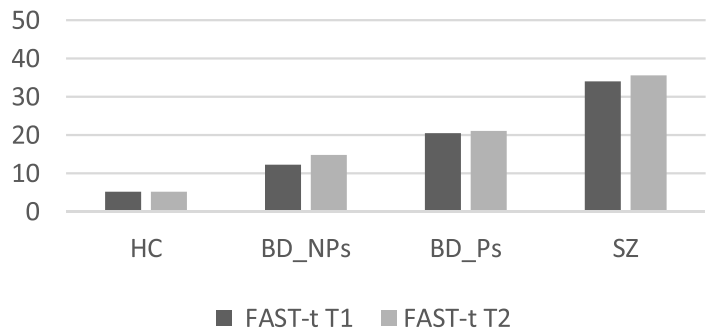

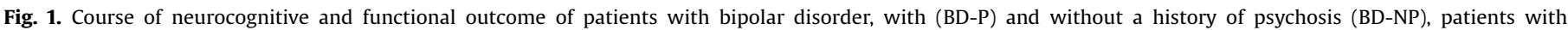

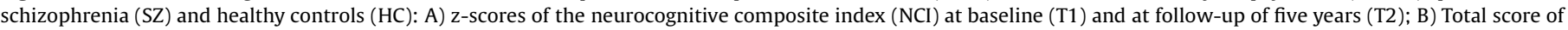
function dimension of the Global Assessment of Functioning (GAF-F); C) Total score of the Functioning Assessment Short Test (FAST).

Table 4

Repeated measures analysis of variance of patients and healthy controls for neurocognitive and functional measures. Significance of test post-hoc (Bonferroni test).

\begin{tabular}{|c|c|c|c|c|c|c|}
\hline & BD-NP vs HC & BD-P vs HC & SZ vs HC & BD-NP vs BD-P & BD-NP vs SZ & BD-P vs SZ \\
\hline NCI & .002 & .000 & .000 & NS & NS & NS \\
\hline Speed processing index & .001 & .000 & .000 & NS & NS & NS \\
\hline TMT-A & NS & .040 & .030 & NS & NS & NS \\
\hline Digit-symbol coding & .001 & .000 & .000 & NS & NS & NS \\
\hline Verbal fluency (categ) & NS & .000 & .000 & NS & NS & NS \\
\hline Working memory index & NS & .006 & .001 & NS & .005 & NS \\
\hline Digit span backward & NS & .035 & .022 & NS & NS & NS \\
\hline Letter-number & NS & .004 & .000 & .007 & .000 & NS \\
\hline Verbal memory index & .025 & .009 & .012 & NS & NS & NS \\
\hline Learning & .015 & .003 & .001 & NS & NS & NS \\
\hline Immediate free recall & .019 & .003 & .004 & NS & NS & NS \\
\hline Delayed free recall & .009 & .005 & .006 & NS & NS & NS \\
\hline Recognition & NS & NS & NS & NS & NS & NS \\
\hline Visual memory index & .001 & .003 & .001 & NS & NS & NS \\
\hline ROCFT immediate recall & .001 & .003 & .000 & NS & NS & NS \\
\hline ROCFT delayed recall & .002 & .004 & .002 & NS & NS & NS \\
\hline Executive function index & .008 & .002 & .000 & NS & NS & NS \\
\hline WCST No. categories & .009 & .031 & .001 & NS & NS & NS \\
\hline WCST perseverative errors & .004 & .010 & .000 & NS & NS & NS \\
\hline TMT-B & .020 & .002 & .001 & NS & NS & NS \\
\hline Verbal fluency (FAS) & NS & NS & NS & NS & NS & NS \\
\hline Stroop & NS & NS & .011 & NS & NS & NS \\
\hline Attention index & NS & .024 & .009 & NS & NS & NS \\
\hline CPT hits & NS & .015 & .027 & NS & NS & NS \\
\hline CPT sensitivity $A^{\prime}$ & NS & NS & .008 & NS & NS & NS \\
\hline GAF-F & NS & .000 & .000 & .049 & .000 & NS \\
\hline FAST total score & NS & .000 & .000 & .033 & .000 & .005 \\
\hline Autonomy & NS & NS & .000 & NS & .000 & .021 \\
\hline Occupational functioning & NS & .000 & .000 & .016 & .000 & NS \\
\hline Cognitive functioning & NS & NS & .000 & NS & .000 & .001 \\
\hline Financial issues & NS & NS & .004 & NS & .026 & NS \\
\hline Interpersonal relationships & NS & .001 & .000 & .010 & .000 & .000 \\
\hline Leisure time & .000 & .000 & .000 & NS & .035 & NS \\
\hline
\end{tabular}

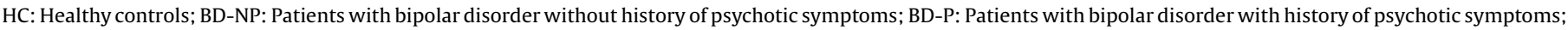

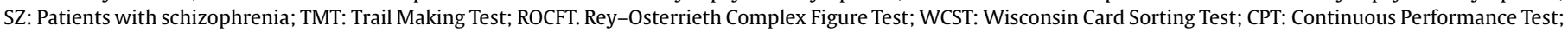
GAF-F: Function dimension of the Global Assessment of Functioning; FAST: Functioning Assessment Short Test.

characteristics, with only slight differences found between them, circumscribed to the working memory domain. Similarly, other authors did not find relevant neurocognitive differences between these two subsets of patients [24,27,62-64], even in early stages BD
[44,65], although there exist some discrepant findings [18,66]. In any case, there is broad agreement on the presence of an overlapping in the neurocognitive performance of both groups of patients in a way that the differences found are subtle [18]. On 
Table 5

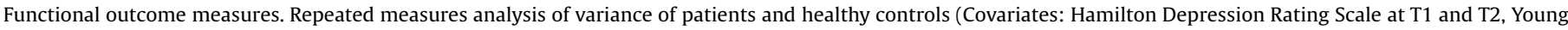
Mania rating scale at $\mathrm{T} 1$ ).

\begin{tabular}{|c|c|c|c|c|c|c|c|c|c|c|c|}
\hline & \multicolumn{4}{|l|}{$\mathrm{T} 1$} & \multicolumn{4}{|l|}{$\mathrm{T} 2$} & \multirow{2}{*}{$\begin{array}{l}\text { Effect of } \\
\text { group }\end{array}$} & \multirow{2}{*}{$\begin{array}{l}\text { Effect of } \\
\text { time }\end{array}$} & \multirow{2}{*}{$\begin{array}{l}\mathrm{G} \times \mathrm{T} \\
\text { Interaction }\end{array}$} \\
\hline & $\begin{array}{l}\mathrm{HC} \\
\mathrm{N}=36\end{array}$ & $\begin{array}{l}\text { BD_NPs } \\
\mathrm{N}=34\end{array}$ & $\begin{array}{l}\text { BD_Ps } \\
\mathrm{N}=44\end{array}$ & $\begin{array}{l}\mathrm{SZ} \\
\mathrm{N}=35\end{array}$ & $\begin{array}{l}\mathrm{HC} \\
\mathrm{N}=36\end{array}$ & $\begin{array}{l}\text { BD_NPs } \\
\mathrm{N}=34\end{array}$ & $\begin{array}{l}\text { BD_Ps } \\
\mathrm{N}=44\end{array}$ & $\begin{array}{l}\mathrm{SZ} \\
\mathrm{N}=35\end{array}$ & & & \\
\hline GAF-F & $\begin{array}{l}91.9 \\
(6.0)\end{array}$ & $\begin{array}{l}75.3 \\
(20.0)\end{array}$ & $\begin{array}{l}64.2 \\
(23.7)\end{array}$ & $\begin{array}{l}48.9 \\
(19.8)\end{array}$ & $\begin{array}{l}89.5 \\
(5.1)\end{array}$ & $\begin{array}{l}71.5 \\
(22.8)\end{array}$ & $\begin{array}{l}62.4 \\
(24.7)\end{array}$ & $\begin{array}{l}46.5 \\
(20.2)\end{array}$ & $0.000^{1}$ & 0.001 & 0.518 \\
\hline FAST total score & $5.2(3.6)$ & $\begin{array}{l}12.4 \\
(12.8)\end{array}$ & $\begin{array}{l}20.5 \\
(15.5)\end{array}$ & $\begin{array}{l}34.1 \\
(17.0)\end{array}$ & $5.2(4.0)$ & $\begin{array}{l}14.8 \\
(15.6)\end{array}$ & $21.1(15.2)$ & $\begin{array}{l}35.6 \\
(17.5)\end{array}$ & $0.000^{1}$ & 0.334 & 0.511 \\
\hline Autonomy & $0.1(0.2)$ & $1.1(1.9)$ & $2.1(3.1)$ & $4.5(3.8)$ & $0.1(0.4)$ & $2.1(3.1)$ & $2.1(3.1)$ & $5.7(6.9)$ & $0.000^{2}$ & 0.229 & 0.744 \\
\hline Occupational functioning & $2.3(2.7)$ & $3.9(5.5)$ & $7.9(6.5)$ & $11.3(5.4)$ & $2.5(2.8)$ & $5.7(6.7)$ & $8.1(6.5)$ & $12.2(5.3)$ & $0.000^{3}$ & 0.167 & 0.216 \\
\hline Cognitive functioning & $0.3(0.7)$ & $1.5(2.6)$ & $1.8(2.3)$ & $4.1(3.1)$ & $0.3(0.7)$ & $1.6(2.7)$ & $1.7(2.0)$ & $4.1(3.3)$ & $0.000^{2}$ & 0.087 & 0.819 \\
\hline Financial issues & $0.0(0.1)$ & $0.4(0.89)$ & $0.9(1.6)$ & $1.9(2.0)$ & $0.0(0.1)$ & $0.8(1.6)$ & $1.2(2.0)$ & $2.0(2.1)$ & $0.003^{4}$ & 0.817 & 0.248 \\
\hline $\begin{array}{l}\text { Interpersonal } \\
\text { relationships }\end{array}$ & $1.0(1.1)$ & $2.3(3.1)$ & $4.2(4.1)$ & $8.1(3.8)$ & $1.2(1.0)$ & $2.1(2.5)$ & $4.4(4.1)$ & $7.7(4.0)$ & $0.000^{1}$ & 0.084 & 0.124 \\
\hline Leisure time & $1.6(1.3)$ & $3.4(1.1)$ & $3.7(1.3)$ & $4.3(1.8)$ & $1.4(1.1)$ & $3.4(1.4)$ & $3.7(1.3)$ & $4.4(2.1)$ & $0.000^{5}$ & 0.398 & 0.969 \\
\hline
\end{tabular}

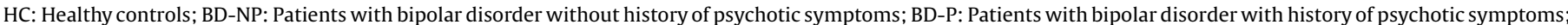

SZ: Patients with schizophrenia; GAF-F: Function dimension of the Global Assessment of Functioning; FAST: Functioning Assessment Short Test.

1 HC, BD-NP $>$ BD-P $>$ SZ.

2 HC, BD-NP, BD-P $>$ SZ.

${ }^{3} \mathrm{HC}, \mathrm{BD}-\mathrm{NP}>\mathrm{BD}-\mathrm{P}, \mathrm{SZ}$

${ }^{4} \mathrm{HC}>\mathrm{SZ}$.

5 HC > BD-NP, BD-P, SZ

the other hand, also a number of studies have found the presence of differences in the working memory domain [12,18,67-70], although the effect size is small. Interestingly, in our study, BDNP did not differ from the control group. In this regard, it has been suggested that working memory deficits found in patients with BD-P could indicate the presence of a possible association between psychosis and frontal lobe dysfunction [18].

Third, considering psychosocial functioning, while patients with BD-NP have a level of performance similar to that found in HC, BD-P had a worse performance than $\mathrm{HC}$ on most functional measures. Likewise, as has been found in other studies, BD-P showed lower impairment than SZ in overall functioning measures [71,72]. In addition, BD-P had a worse level of psychosocial functioning than BD-NP, in a way that we could not replicate the findings of our previous cross-sectional study [29]. However, the present results are in accordance with other authors which also reported that patients with BD-P had a worse functional performance than patients with BD-NP [20,40-43]. Therefore, the psychosocial functioning of BD-P would occupy an intermediate position between the functional impairment of patients with BD-NP and that of patients with SZ [23].

The current study has several limitations that should be acknowledged. Most importantly, the small sample size. Another limitation of the study is that all patients were receiving pharmacological treatment. It should be noted that a higher percentage of patients with BD-P were being treated with antipsychotics. Thus, the possibility of a deleterious effect of antipsychotics on the level of neurocognitive and psychosocial functioning cannot be ruled out $[73,74]$. Finally, a Cronbach alpha coefficient for the working memory domain of 0.585 could alert about the appropriateness of the instruments used for measuring this domain. However, this theoretically low value should be interpreted cautiously, since it is a coefficient which depends on the number of items (only two in our case, WAIS-III digit span backward subtest and letter-number sequencing subtest of the Wechsler Memory Scale), and the variation of the population where consistency is evaluated, in such a way that higher values of alpha are expected in homogeneous populations, and our sample included patients with $\mathrm{BD}$, patients with SZ and control individuals [75].

Despite the mentioned limitations, we should also note several strengths. First the longitudinal design. Second, the 5-year duration of the follow-up, which could be considered long enough to detect changes. Third, the inclusion of a healthy control group which was also assessed after a five-year follow-up. Fourth, the fact that both patients with BD and patients with SZ were in symptomatic remission at the two time-points of assessment.

In conclusion, our study highlighted that the course of the neurocognitive functioning and the functional outcome in both patients with schizophrenia and patients with bipolar disorder, including those with a history of psychotic symptoms, is stable. Likewise, the profile of neurocognitive impairment of patients with schizophrenia or bipolar disorder, with or without psychotic symptoms, is similar, with only quantitative differences circumscribed to certain domains, such as working memory. Importantly, the results suggest the existence of a subgroup of patients with bipolar disorder, characterized by the absence of psychotic symptoms, which does not show a functional deterioration. Nevertheless, these results should be considered preliminary, being necessary longitudinal studies with larger samples and a longer duration to confirm these findings.

\section{Disclosure of interest}

Dr. R. Rodriguez-Jimenez has been a consultant for, spoken in activities of, or received grants from: Instituto de Salud Carlos III, Fondo de Investigación Sanitaria (FIS),Centro de Investigación Biomédica en Red de Salud Mental (CIBERSAM), Madrid Regional Government (S2010/BMD-2422 AGES; B2017/BMD-3740 AGES CM 2-CM), JanssenCilag, Lundbeck, Otsuka, Pfizer, Ferrer, Juste, Takeda. Dr. Vieta has received grants and served as consultant, advisor or CME speaker for the following entities: AB-Biotics, Allergan, Angelini, AstraZeneca, Bristol-Myers Squibb, Dainippon Sumitomo Pharma, Farmindustria, Ferrer, Forest Research Institute, Gedeon Richter, Glaxo-Smith-Kline, Janssen, Lundbeck, Otsuka, Pfizer, Roche, Sanofi-Aventis, Servier, Shire, Sunovion, Takeda, the Brain and Behaviour Foundation, the Spanish Ministry of Science and Innovation (CIBERSAM), the Seventh European Framework Programme (ENBREC), and the Stanley Medical Research Institute. All other authors declare that they have no conflicts of interest.

\section{Acknowledgements}

This work was supported in part by the grant PI16/00359 (Fondo de Investigaciones Sanitarias, FIS, and FEDER), by Madrid Regional Government (R\&D activities in Biomedicine S2017/BMD3740 (AGES-CM 2-CM)) and Structural Funds of the European 
Union, and by the Centro de Investigación Biomédica en Red de Salud Mental (CIBERSAM) of the Instituto de Salud Carlos III.

\section{Appendix A. Supplementary data}

Supplementary data associated with this article can be found, in the online version, at https://doi.org/10.1016/j.eurpsy.2018.11.008.

\section{References}

[1] Bora E, Yucel M, Pantelis C. Cognitive endophenotypes of bipolar disorder: a meta-analysis of neuropsychological deficits in euthymic patients and their first-degree relatives. J Affect Disord 2009;113:1-20, doi:http://dx.doi.org/ 10.1016/j.jad.2008.06.009.

[2] Bora E, Özerdem A. Meta-analysis of longitudinal studies of cognition in bipolar disorder: comparison with healthy controls and schizophrenia. Psychol Med 2017;47:2753-66, doi:http://dx.doi.org/10.1017/ S0033291717001490.

[3] Huxley N, Baldessarini RJ. Disability and its treatment in bipolar disorde patients. Bipolar Disord 2007:9:183-96, doi:http://dx.doi.org/10.1111/j.13995618.2007.00430.x.

[4] Gitlin MJ, Miklowitz DJ. The difficult lives of individuals with bipolar disorder: a review of functional outcomes and their implications for treatment. J Affect Disord 2017;209:147-54, doi:http://dx.doi.org/10.1016/j.jad.2016.11.021.

[5] Goldberg JF, Harrow M, Grossman LS. Course and outcome in bipolar affective disorder: a longitudinal follow- up study. Am J Psychiatry 1995;152:379-84, doi:http://dx.doi.org/10.1176/ajp.152.3.379.

[6] Bauer M, Andreassen OA, Geddes JR, Vedel Kessing L, Lewitzka U, Schulze TG, et al. Areas of uncertainties and unmet needs in bipolar disorders: clinical and research perspectives. Lancet Psychiatry 2018, doi:http://dx.doi.org/10.1016/ S2215-0366(18)30253-0.

[7] Roux P, Raust A, Cannavo A-S, Aubin V, Aouizerate B, Azorin J-M, et al. Associations between residual depressive symptoms, cognition, and functioning in patients with euthymic bipolar disorder: results from the FACEBD cohort. Br J Psychiatry 2017;211:381-7, doi:http://dx.doi.org/10.1192/bjp. bp.117.201335.

[8] Jiménez E, Solé B, Arias B, Mitjans M, Varo C, Reinares M, et al. Characterizing decision-making and reward processing in bipolar disorder: a cluster analysis. Eur Neuropsychopharmacol 2018;28:863-74, doi:http://dx.doi.org/10.1016/j. euroneuro.2018.04.001.

[9] Reinares M, Papachristou E, Harvey P, Mar Bonnín C, Sánchez-Moreno J, Torrent C, et al. Towards a clinical staging for bipolar disorder: defining patien subtypes based on functional outcome. J Affect Disord 2013;144:65-71, doi: http://dx.doi.org/10.1016/j.jad.2012.06.005.

[10] Solé B, Bonnin CM, Jiménez E, Torrent C, Torres I, Varo C, et al. Heterogeneity of functional outcomes in patients with bipolar disorder: a cluster-analytic approach. Acta Psychiatr Scand 2018, doi:http://dx.doi.org/10.1111/acps.12871.

[11] Burdick KE, Russo M, Frangou S, Mahon K, Braga RJ, Shanahan M, et al Empirical evidence for discrete neurocognitive subgroups in bipolar disorder: clinical implications. Psychol Med 2014;44:3083-96, doi:http://dx.doi.org/ 10.1017/S0033291714000439.

[12] Simonsen C, Sundet K, Vaskinn A, Birkenaes AB, Engh JA, Faerden A, et al. Neurocognitive dysfunction in bipolar and schizophrenia spectrum disorders depends on history of psychosis rather than diagnostic group. Schizophr Bull 2011;37:73-83, doi:http://dx.doi.org/10.1093/schbul/sbp034.

[13] Latalova K, Prasko J, Diveky T, Velartova H. Cognitive impairment in bipolar disorder. Biomed Pap Med Fac Univ Palacky Olomouc Czech Repub 2011;155:19-26.

[14] Tohen M, Zarate CA, Hennen J, H-MK Khalsa, Strakowski SM, Gebre-Medhin P et al. The McLean-Harvard First-Episode Mania Study: prediction of recovery and first recurrence. Am J Psychiatry 2003;160:2099-107, doi:http://dx.doi. org/10.1176/appi.ajp.160.12.2099.

[15] Green MF, Harvey PD. Cognition in schizophrenia: past, present, and future Schizophr Res Cogn 2014;1:e1-9, doi:http://dx.doi.org/10.1016/j. scog.2014.02.001.

[16] Green MF. Impact of cognitive and social cognitive impairment on functiona outcomes in patients with schizophrenia. J Clin Psychiatry 2016;77(Suppl. 2):8-11, doi:http://dx.doi.org/10.4088/JCP.14074su1c.02.

[17] Bipolar Disorder and Schizophrenia Working Group of the Psychiatric Genomics Consortium, Bipolar Disorder and Schizophrenia Working Group of the Psychiatric Genomics Consortium S, McQuillin A, Boocock J, Stahl EA Pavlides JMW, et al. Genomic dissection of bipolar disorder and schizophrenia, including 28 subphenotypes. Cell 2018;173:, doi:http://dx.doi.org/10.1016/j. cell.2018.05.046 1705-1715.e16.

[18] Bora E, Yücel M, Pantelis C. Neurocognitive markers of psychosis in bipola disorder: a meta-analytic study. J Affect Disord 2010;127:1-9, doi:http://dx. doi.org/10.1016/j.jad.2010.02.117.

[19] Buoli M, Caldiroli A, Cumerlato Melter C, Serati M, de Nijs J, Altamura AC Biological aspects and candidate biomarkers for psychotic bipolar disorder: a systematic review. Psychiatry Clin Neurosci 2016;70:227-44, doi:http://dx. doi.org/10.1111/pcn.12386

[20] Dell'Osso B, Camuri G, Cremaschi L, Dobrea C, Buoli M, Ketter TA, et al Lifetime presence of psychotic symptoms in bipolar disorder is associated with less favorable socio-demographic and certain clinical features. Compr Psychiatry 2017;76:169-76, doi:http://dx.doi.org/10.1016/j. comppsych.2017.04.005.

[21] MacQueen GM, Young LT, Joffe RT. A review of psychosocial outcome in patients with bipolar disorder. Acta Psychiatr Scand 2001;103:163-70.

[22] Brissos S, Dias VV, Soeiro-de-Souza MG, Balanzá-Martínez V, Kapczinski F. The impact of a history of psychotic symptoms on cognitive function in euthymic bipolar patients: a comparison with schizophrenic patients and healthy controls. Rev Bras Psiquiatr 2011;33:353-61.

[23] Szoke A, Meary A, Trandafir A, Bellivier F, Roy I, Schurhoff F, et al. Executive deficits in psychotic and bipolar disorders - implications for our understanding of schizoaffective disorder. Eur Psychiatry 2008;23:20-5, doi:http://dx.doi. org/10.1016/j.eurpsy.2007.10.006.

[24] Savitz J, van der Merwe L, Stein DJ, Solms M, Ramesar R. Neuropsychological status of bipolar I disorder: impact of psychosis. Br J Psychiatry 2009;194:24351, doi:http://dx.doi.org/10.1192/bjp.bp.108.052001.

[25] Dickerson FB, Boronow JJ, Stallings CR, Origoni AE, Cole S, Yolken RH. Association between cognitive functioning and employment status of persons with bipolar disorder. Psychiatr Serv 2004;55:54-8, doi:http://dx.doi.org/ 10.1176/appi.ps.55.1.54

[26] Soni A, Singh P, Shah R, Bagotia S. Impact of cognition and clinical factors on functional outcome in patients with bipolar disorder. East Asian Arch Psychiatry 2017;27:26-34.

[27] Sánchez-Morla EM, Barabash A, Martínez-Vizcaíno V, Tabarés-Seisdedos R, Balanzá-Martínez V, Cabranes-Díaz JA, et al. Comparative study of neurocognitive function in euthymic bipolar patients and stabilized schizophrenic patients. Psychiatry Res 2009;169:220-8, doi:http://dx.doi.org/ 10.1016/j.psychres.2008.06.032.

[28] Jiménez-López E, Aparicio AI, Sánchez-Morla EM, Rodriguez-Jimenez R, Vieta E, Santos JL. Neurocognition in patients with psychotic and non-psychotic bipolar I disorder. A comparative study with individuals with schizophrenia. J Affect Disord 2017;222:169-76, doi:http://dx.doi.org/10.1016/j. jad.2017.07.014.

[29] Jiménez-López E, Sánchez-Morla EM, Aparicio AI, López-Villarreal A, Martínez-Vizcaíno V, Rodriguez-Jimenez R, et al. Psychosocial functioning in patients with psychotic and non-psychotic bipolar I disorder. A comparative study with individuals with schizophrenia. J Affect Disord 2018;229:, doi: http://dx.doi.org/10.1016/j.jad.2017.12.094.

[30] Caldieraro MA, Sylvia LG, Dufour S, Walsh S, Janos J, Rabideau DJ, et al. Clinical correlates of acute bipolar depressive episode with psychosis. J Affect Disord 2017;217:29-33, doi:http://dx.doi.org/10.1016/j.jad.2017.03.059.

[31] Santos JL, Aparicio A, Bagney A, Sánchez-Morla EM, Rodríguez-Jiménez R, Mateo J, et al. A five-year follow-up study of neurocognitive functioning in bipolar disorder. Bipolar Disord 2014;16:722-31, doi:http://dx.doi.org/ 10.1111/bdi.12215.

[32] Mora E, Portella MJ, Forcada I, Vieta E, Mur M. Persistence of cognitive impairment and its negative impact on psychosocial functioning in lithiumtreated, euthymic bipolar patients: a 6-year follow-up study. Psychol Med 2013;43:1187-96, doi:http://dx.doi.org/10.1017/S0033291712001948.

[33] Tabarés-Seisdedos R, Balanzá-Martínez V, Sánchez-Moreno J, Martinez-Aran A, Salazar-Fraile J, Selva-Vera G, et al. Neurocognitive and clinical predictors of functional outcome in patients with schizophrenia and bipolar I disorder at one-year follow-up. J Affect Disord 2008;109:286-99, doi:http://dx.doi.org/ 10.1016/j.jad.2007.12.234

[34] Demmo C, Lagerberg TV, Kvitland LR, Aminoff SR, Hellvin T, Simonsen C, et al. Neurocognitive functioning, clinical course and functional outcome in firsttreatment bipolar I disorder patients with and without clinical relapse: a 1 year follow-up study. Bipolar Disord 2018;20:228-37, doi:http://dx.doi.org/ $10.1111 /$ bdi.12569.

[35] Martino DJ, Igoa A, Scápola M, Marengo E, Samamé C, Strejilevich SA Functional outcome in the middle course of bipolar disorder. J Nerv Ment Dis 2017;205:203-6, doi:http://dx.doi.org/10.1097/NMD.0000000000000583.

[36] Robinson LJ, Ferrier IN. Evolution of cognitive impairment in bipolar disorder: a systematic review of cross-sectional evidence. Bipolar Disord 2006:8:10316, doi:http://dx.doi.org/10.1111/j.1399-5618.2006.00277.x.

[37] Rosa AR, Magalhães PVS, Czepielewski L, Sulzbach MV, Goi PD, Vieta E, et al. Clinical staging in bipolar disorder: focus on cognition and functioning. J Clin Psychiatry 2014, doi:http://dx.doi.org/10.4088/JCP.13m08625.

[38] López-Jaramillo C, Lopera-Vásquez J, Gallo A, Ospina-Duque J, Bell V, Torrent C, et al. Effects of recurrence on the cognitive performance of patients with bipolar I disorder: implications for relapse prevention and treatment adherence. Bipolar Disord 2010;12:557-67, doi:http://dx.doi.org/10.1111/ j.1399-5618.2010.00835.x.

[39] Sánchez-Morla EM, López-Villarreal A Jiménez-López E Aparicio AI Martínez-Vizcaíno V, Roberto R-J, et al. Impact of number of episodes on neurocognitive trajectory in bipolar disorder patients: a 5-year follow-up study. Psychol Med 2018;1-9, doi:http://dx.doi.org/10.1017/ S0033291718001885.

[40] Levy B, Medina AM, Weiss RD. Cognitive and psychosocial functioning in bipolar disorder with and without psychosis during early remission from an acute mood episode: a comparative longitudinal study. Compr Psychiatry 2013;54:618-26, doi:http://dx.doi.org/10.1016/j. comppsych.2012.12.018.

[41] Canuso CM, Bossie CA, Zhu Y, Youssef E, Dunner DL. Psychotic symptoms in patients with bipolar mania. J Affect Disord 2008;111:164-9, doi:http://dx.doi. org/10.1016/j.jad.2008.02.014. 
[42] Goes FS, Sadler B, Toolan J, Zamoiski RD, Mondimore FM, MacKinnon DF, et al Psychotic features in bipolar and unipolar depression. Bipolar Disord 2007;9:901-6, doi:http://dx.doi.org/10.1111/j.1399-5618.2007.00460.x.

[43] Kempf L, Hussain N, Potash JB. Mood disorder with psychotic features, schizoaffective disorder, and schizophrenia with mood features: trouble at the borders. Int Rev Psychiatry 2005;17:9-19, doi:http://dx.doi.org/10.1080/ 09540260500064959.

[44] Trisha C, Golnoush A, Jan-Marie K, Torres IJ, Yatham LN. Cognitive functioning in first episode bipolar I disorder patients with and without history of psychosis. J Affect Disord 2018;227:109-16, doi:http://dx.doi.org/10.1016/j. jad.2017.10.003.

[45] Van Gorp WG, Altshuler L, Theberge DC, Wilkins J, Dixon W. Cognitive impairment in euthymic bipolar patients with and without prior alcohol dependence. A preliminary study. Arch Gen Psychiatry 1998;55:41-6.

[46] Peralta V, Cuesta MJ. Psychometric properties of the positive and negative syndrome scale (PANSS) in schizophrenia. Psychiatry Res 1994;53:31-40.

[47] Ramos-Brieva JA, Cordero-Villafafila A. A new validation of the Hamilton rating scale for depression. J Psychiatr Res 1988;22:21-8.

[48] Colom F, Vieta E, Martínez-Arán A, Garcia-Garcia M, Reinares M, Torrent C, et al. Spanish version of a scale for the assessment of mania: validity and reliability of the Young Mania Rating Scale. Med Clin (Barc) 2002;119:366-71.

[49] Rosa AR, Sánchez-Moreno J, Martínez-Aran A, Salamero M, Torrent C, Reinares $\mathrm{M}$, et al. Validity and reliability of the Functioning Assessment Short Test (FAST) in bipolar disorder. Clin Pract Epidemiol Ment Health 2007;3:5, doi: http://dx.doi.org/10.1186/1745-0179-3-5.

[50] Pedersen G, Hagtvet KA, Karterud S. Generalizability studies of the global assessment of functioning-split version. Compr Psychiatry 2007;48:88-94, doi:http://dx.doi.org/10.1016/j.comppsych.2006.03.008.

[51] Costa LG, Massuda R, Pedrini M, Passos IC, Czepielewski LS, Brietzke E, et al. Functioning in early and late stages of schizophrenia. Trends Psychiatry Psychother 2014;36:209-13, doi:http://dx.doi.org/10.1590/2237-6089-20140028.

[52] González-Ortega I, Rosa A, Alberich S, Barbeito S, Vega P, Echeburúa E, et al. Validation and use of the functioning assessment short test in first psychotic episodes. J Nerv Ment Dis 2010;198:836-40, doi:http://dx.doi.org/10.1097/ NMD.0b013e3181f97bf9.

[53] Nuechterlein KH, Green MF, Kern RS, Baade LE, Barch DM, Cohen JD, et al. The MATRICS consensus cognitive battery, part 1: test selection, reliability, and validity. Am J Psychiatry 2008;165:203-13, doi:http://dx.doi.org/10.1176/appi. ajp.2007.07010042.

[54] Burdick KE, Goldberg JF, Harrow M, Faull RN, Malhotra AK. Neurocognition as a stable endophenotype in bipolar disorder and schizophrenia. J Nerv Ment Dis 2006;194:255-60, doi:http://dx.doi.org/10.1097/01. nmd.0000207360.70337.7e.

[55] Depp CA, Savla GN, Moore DJ, Palmer BW, Stricker JL, Lebowitz BD, et al. Shortterm course of neuropsychological abilities in middle-aged and older adults with bipolar disorder. Bipolar Disord 2008;10:684-90, doi:http://dx.doi.org/ 10.1111/j.1399-5618.2008.00601.x.

[56] SNTM Schouws, Comijs HC, Dols A, Beekman ATF, Stek ML. Five-year follow-up of cognitive impairment in older adults with bipolar disorder. Bipolar Disord 2016;18:148-54, doi:http://dx.doi.org/10.1111/bdi.12374.

[57] Kurtz MM. Neurocognitive impairment across the lifespan in schizophrenia: an update. Schizophr Res 2005;74:15-26, doi:http://dx.doi.org/10.1016/j. schres.2004.07.005.

[58] Heaton RK, Gladsjo JA, Palmer BW, Kuck J, Marcotte TD, Jeste DV. Stability and course of neuropsychological deficits in schizophrenia. Arch Gen Psychiatry 2001;58:24-32.

[59] Hoff AL, Svetina C, Shields G, Stewart J, DeLisi LE. Ten year longitudinal study of neuropsychological functioning subsequent to a first episode of schizophrenia. Schizophr Res 2005;78:27-34, doi:http://dx.doi.org/10.1016/j. schres.2005.05.010.
[60] Velthorst E, A-KJ Fett, Reichenberg A, Perlman G, van Os J, Bromet EJ, et al. The 20 -year longitudinal trajectories of social functioning in individuals with psychotic disorders. Am J Psychiatry 2016, doi:http://dx.doi.org/10.1176/appi. ajp.2016.15111419 appiajp201615111419.

[61] Jobe TH, Harrow M. Long-term outcome of patients with schizophrenia: a review. Can J Psychiatry 2005;50:892-900, doi:http://dx.doi.org/10.1177/ 070674370505001403.

[62] Amann B, Gomar JJ, Ortiz-Gil J, McKenna P, Sans-Sansa B, Sarró S, et al. Executive dysfunction and memory impairment in schizoaffective disorder: a comparison with bipolar disorder, schizophrenia and healthy controls. Psychol Med 2012:42:2127-35, doi:http://dx.doi.org/10.1017/S0033291712000104.

[63] Ancín I, Cabranes JA, Santos JL, Sánchez-Morla E, Barabash A. Executive deficits: a continuum schizophrenia-bipolar disorder or specific to schizophrenia? J Psychiatr Res 2013;47:1564-71, doi:http://dx.doi.org/ 10.1016/j.jpsychires.2013.07.008.

[64] Liu SK, Chiu C-H, Chang C-J, Hwang T-J, Hwu H-G, Chen WJ. Deficits in sustained attention in schizophrenia and affective disorders: stable versus state-dependent markers. Am J Psychiatry 2002;159:975-82, doi:http://dx. doi.org/10.1176/appi.ajp.159.6.975.

[65] Demmo C, Lagerberg TV, Aminoff SR, Hellvin T, Kvitland LR, Simonsen C, et al History of psychosis and previous episodes as potential explanatory factors for neurocognitive impairment in first-treatment bipolar I disorder. Bipolar Disord 2016;18:136-47, doi:http://dx.doi.org/10.1111/bdi.12377.

[66] Krabbendam L, Arts B, van Os J, Aleman A. Cognitive functioning in patients with schizophrenia and bipolar disorder: a quantitative review. Schizophr Res 2005;80:137-49, doi:http://dx.doi.org/10.1016/j.schres.2005.08.004.

[67] Glahn DC, Bearden CE, Cakir S, Barrett JA, Najt P, Serap Monkul E, et al. Differential working memory impairment in bipolar disorder and schizophrenia: effects of lifetime history of psychosis. Bipolar Disord 2006;8:117-23, doi:http://dx.doi.org/10.1111/j.1399-5618.2006.00296.x.

[68] Glahn DC, Bearden CE, Barguil M, Barrett J, Reichenberg A, Bowden CL, et al. The neurocognitive signature of psychotic bipolar disorder. Biol Psychiatry 2007;62:910-6, doi:http://dx.doi.org/10.1016/j.biopsych.2007.02.001.

[69] Bora E. Neurocognitive features in clinical subgroups of bipolar disorder: a meta-analysis. J Affect Disord 2018;229:125-34, doi:http://dx.doi.org/10.1016/ j.jad.2017.12.057.

[70] Hewedi D, Frydecka D, Moustafa A. Impairments of working memory in schizophrenia and bipolar disorder: the effect of history of psychotic symptoms and different aspects of cognitive task demands. Eur Psychiatry 2015;30:596, doi:http://dx.doi.org/10.1016/S0924-9338(15)30475-2.

[71] Lewandowski K, Cohen BM, Keshavan MS, Sperry SH, Öngür D. Neuropsychological functioning predicts community outcomes in affective and non-affective psychoses: a 6-month follow-up. Schizophr Res 2013;148:34-7, doi:http://dx.doi.org/10.1016/j.schres.2013.05.012.

[72] Dickerson FB, Sommerville J, Origoni AE, Ringel NB, Parente F. Outpatients with schizophrenia and bipolar I disorder: Do they differ in their cognitive and social functioning? Psychiatry Res 2001;102:21-7.

[73] Kim J-H, Son Y-D, Kim H-K, Lee S-Y, Cho S-E, Kim Y-B, et al. Antipsychoticassociated mental side effects and their relationship to dopamine D2 recepto occupancy in striatal subdivisions. J Clin Psychopharmacol 2011:31:507-11, doi:http://dx.doi.org/10.1097/JCP.0b013e318222353a.

[74] Wunderink L, Nieboer RM, Wiersma D, Sytema S, Nienhuis FJ. Recovery in remitted first-episode psychosis at 7 years of follow-up of an early dose reduction/discontinuation or maintenance treatment strategy. JAMA Psychiatry 2013;70:913, doi:http://dx.doi.org/10.1001/ jamapsychiatry.2013.19.

[75] De Vet HC, Terwee CB, Mokkink LB, Dirk LK. Measurement in medicine: a practical guide (Practical guides to biostatistics and epidemiology). Cambridge: Cambridge University Press; 2011. 